\title{
Estimating the Motion of the LAD: A Simulation-Based Study
}

\author{
I.A. Kakadiaris ${ }^{1 \star}$, A. Pednekar ${ }^{1}$, G. Zouridakis $^{2}$, and K. Grigoriadis ${ }^{3}$ \\ 1 Dept. of Computer Science, Univ. of Houston, Houston TX 77204-3010, USA \\ 2 Dept. of Neuroscience, Univ. of Texas-Medical School, Houston TX 77030, USA \\ 3 Dept. of Mechanical Engineering, Univ. of Houston, Houston TX 77204-4792, USA
}

\begin{abstract}
In this paper, we present a simulation-based study of a motion analysis technique designed to intra-operatively estimate the motion parameters of the LAD coronary artery from endoscopic images.
\end{abstract}

\section{Introduction}

The epicardial coronary arteries exhibit substantial phasic motion during the cardiac cycle. Our goal is to explore the feasibility of intra-operatively tracking the motion of the Left Anterior Descending (LAD) coronary artery using IR endoscopic images and patient-specific pre-operative data. Reconstruction of the three-dimensional geometry of the coronary arteries (e.g., from biplane projection data 1]) has received extensive attention. However, no parametric model of the arterial lumen that captures both the global and local shape characteristics has been presented. Similarly, although there is a substantial body of work on vessel tracking 25, most of the methods are limited to two-dimensional tracking in an image and there is no parametric model of the motion of the LAD during the cardiac cycle.

\section{Methods}

Modeling the arterial lumen: In our simulation-based study, we start with a triangular mesh of the inner lumen of the LDA. Our first objective is to obtain a parametric model of this mesh. To that end we employ the deformable model framework 43] to fit the model to the data. As a geometric model of the LAD we use a deformable model $\mathbf{s}(u, v)$ with a curved axis $\mathbf{e}(u)=\left(e_{1}(u), e_{2},(u), e_{3}(u)\right)^{\top}$ as follows: $\quad \mathbf{s}(u, v)=\left(\begin{array}{c}s_{1}(u, v) \\ s_{2}(u, v) \\ s_{3}(u, v)\end{array}\right)=\left(\begin{array}{l}e_{1}(u)+a_{1}(u) \cos (v) \\ e_{2}(u)+a_{2}(u) \sin (v) \\ e_{3}(u)\end{array}\right)$, where $-\frac{\pi}{2} \leq u \leq \frac{\pi}{2},-\pi \leq v \leq \pi$, and $a_{1}, a_{2} \geq 0$ are the parameters that define the superquadric size in the $\mathrm{x}$ and $\mathrm{y}$ directions, respectively. To capture local deformations we use the finite element method and we represent the deformable model

\footnotetext{
* This material is based in part upon work supported by the Texas Advanced Research Program under Grant No. 003652-0010-1999.
} 
in the form of weighted sums of local polynomial basis functions [4]. Once fitting is accomplished, the estimated values are the parameters of a (pre-operatively obtained) parametric deformable model of the LAD.

Modeling LAD's motion: Our second objective is to describe parametrically the movement of the LAD during the cardiac cycle. For this purpose we obtained, from our colleagues at the Texas Heart Institute, surface meshes of the LAD during five steps of the cardiac cycle. We model the movement of the LAD (due to the heart movement) as a systole/diastole and twist around the major axis of the heart (in this paper, we will not consider the respiratory motion). Let $\mathbf{m}(u, v, t)=\left(m_{1}, m_{2}, m_{3}\right)^{\top}$ be the position of the LAD over time obtained by applying a systole/diastole transformation along with a twist transformation to the initial LAD shape, as described below. Also, let the initial shape $\mathbf{m}(u, v, 0)=\mathbf{s}(u, v)$. The systole/diastole deformation can be parameterized as follows: $\mathbf{o}(u, v, t)=\left(\begin{array}{l}d^{1}(u, t) \\ d^{2}(u, t) \\ d^{3}(u, t)\end{array}\right) \mathbf{s}(u, v)$. Given the above primitive $\mathbf{o}=$ $\left(o_{1}, o_{2}, o_{3}\right)^{\top}$, the parameterized twisting results in a new position for the LAD given by:

$\mathbf{m}(u, v, t)=\left(\begin{array}{l}o_{1} \cos (w(u, t))-o_{2} \sin (w(u, t)) \\ o_{1} \sin (w(u, t))+o_{2} \cos (w(u, t)) \\ o_{3}\end{array}\right)$, where $w(u, t)$ is the time-varying twisting parameter function along the axis of the heart model.

Model-Based Tracking: Our third objective is to track the motion of the LAD using IR endoscopic images. In this paper, we employ simulated IR endoscopic images. We have developed a model-based tracking technique to estimate the motion of the LAD from IR endoscopic images. The central idea of a modelbased tracking approach is the following [3]: Using a previously acquired model of the object that you want to track, for each timestep find the position and orientation of the object such that it produces data like the ones acquired. We use as input the $2 \mathrm{D}$ coordinates of the silhouette of the LAD as computed by segmenting the IR images. The output is the functions: $d^{1}(u, t), d^{2}(u, t), d^{3}(u, t)$, and $w(u, t)$.

\section{Results}

Concerning modeling the shape of the LAD, Fig. 1(a) depicts the estimated deformable model, Fig. 1(b) depicts the estimated axis e $(u)$, and Fig. 1(c) depicts the variation of the radius of the arterial lumen $a_{1}(u)$. Concerning our tracking technique, the input to our algorithm was the two-dimensional data of the silhouette of the LAD during a cardiac cycle obtained from simulated IR endoscopic images. Figs. 1](d,e) depict five samples of the estimated position of the LAD over time. The view chosen for Fig. 1(d) clearly shows the systole/diastole of the heart and the view chosen for Fig. I(e) demonstrates the recovered twisting motion. Figs. 1 (f-h) depict the values of the estimated motion parameters. 


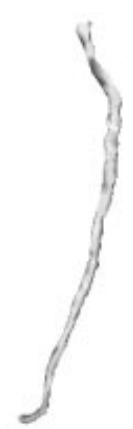

(a)

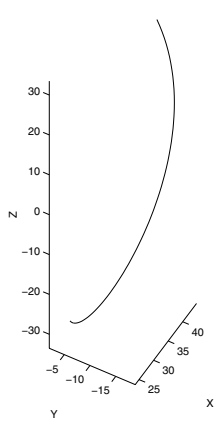

(b)

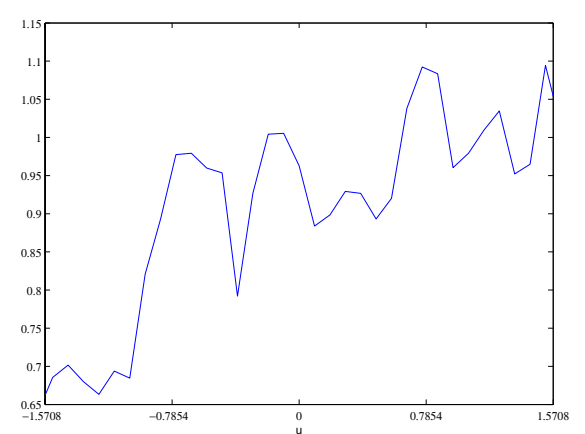

(c)

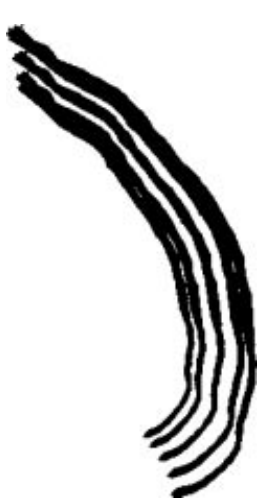

(d)

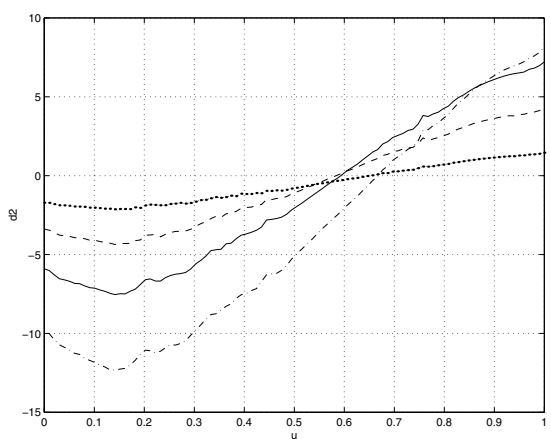

(g)

(e)
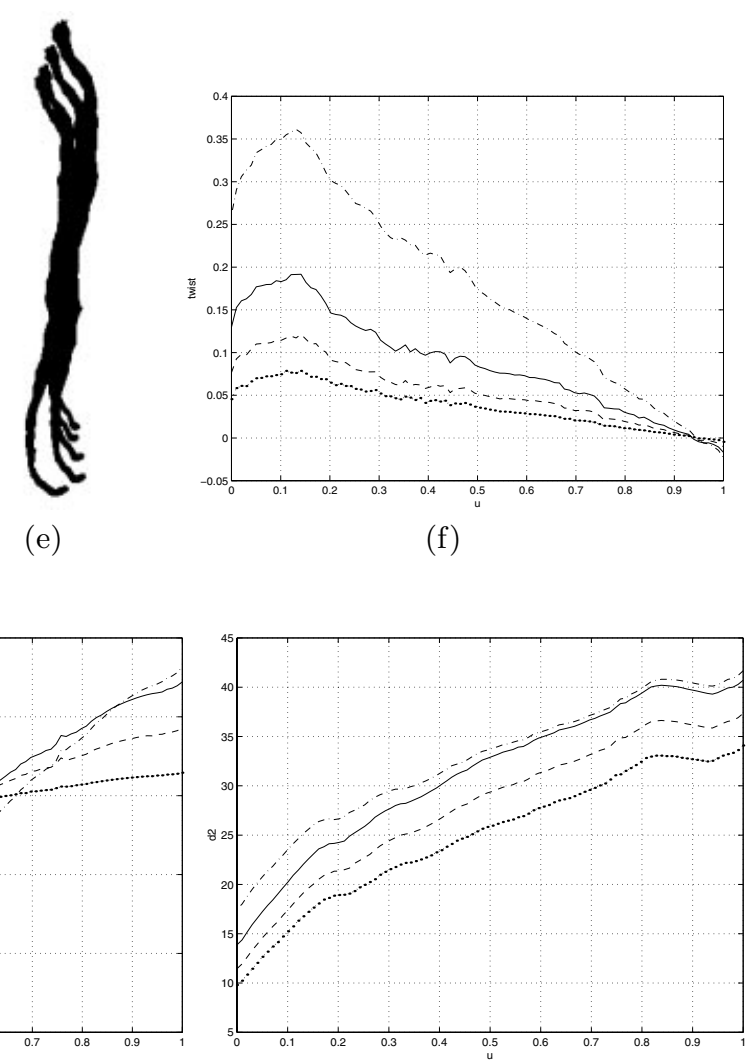

(h)

Fig. 1. (a) Deformable model of the LAD, (b) estimated axis, and (c) estimated radius $a_{1}(u)$. (d,e) Views of the estimated position of the LAD over time, (f-h) Estimated motion parameters $w(u, t), d^{1}(u, t)$ and $d^{2}(u, t)$. 


\section{References}

1. S.-Y.J. Chen and J.D. Carroll. Computer-assisted coronary intervention by use of on-line 3D reconstruction and optimal view strategy. In Medical Image Computing and Computer-Assisted Intervention, 1496, pp. 377-385, Cambridge, MA, 1998.

2. Z. Ding and M.H. Friedman. Dynamics of human coronary arterial motion and its potential role in coronary atherogenesis. J. of Biomech. Engr., 122(5):488, 2000.

3. I.A. Kakadiaris and D. Metaxas. Model-based estimation of $3 \mathrm{D}$ human motion. IEEE Transactions on PAMI, 22(12):1453-1459, 2000.

4. D. Metaxas and D. Terzopoulos. Shape and nonrigid motion estimation through physics-based synthesis. IEEE Transactions on PAMI, 15(6):580 - 591, June 1993.

5. M.S. Sussman, A.B. Kerry, J.M. Pauly, N. Merchant, and G.A. Wright. Tracking the motion of the coronary arteries with the correlation coefficient. In Proceedings of the 7th Scientific Meeting 85 Exhibition of the International Society for Magnetic Resonance in Medicine, Philadelphia, Pennsylvania, 24-28 May 1999. 